Research report

\title{
Quinine sensitivity influences the acceptance of sea-buckthorn and grapefruit juices in 9- to 11-year-old children ${ }^{\text {th }}$
}

\author{
Ditte Hartvig $^{\mathrm{a}, *}$, Helene Hausner ${ }^{\mathrm{a}}$, Karin Wendin ${ }^{\mathrm{a}, \mathrm{b}, \mathrm{c}}$, Wender L.P. Bredie ${ }^{\mathrm{a}}$ \\ a Department of Food Science, Section for Sensory and Consumer Science, Faculty of Science, University of Copenhagen, DK-1958 Frederiksberg C, Denmark \\ ${ }^{\mathrm{b}}$ SP Technical Research Institute of Sweden, IDEON, SE-22370 Lund, Sweden \\ ${ }^{\mathrm{c}}$ Food and Meal Science, Kristianstad University, 29188 Kristianstad, Sweden
}

\section{A R T I C L E I N F O}

\section{Article history:}

Received 6 February 2013

Received in revised form 8 November 2013

Accepted 15 November 2013

Available online 28 November 2013

\section{Keywords:}

Taste sensitivity

Quinine

Bitter foods

Sweet foods

Food acceptance

Nordic foods

\begin{abstract}
A B S T R A C T
The acceptance of novel foods by children is related to a number of factors, and differences in taste sensitivity may form some specific challenges. High sensitivity might be a barrier to the acceptance of sour/ bitter products by children. This study investigated the effect of sensitivity to bitter, sour, sweet, and salty tastes on the acceptance of Nordic juices in 9- to 11-year-old children. A total of 328 children were subjected to two taste sensitivity tests for quinine, citric acid, sucrose, and $\mathrm{NaCl}$. Their acceptance of six juices (carrot, rosehip, sea-buckthorn, lingonberry, grapefruit, and aronia) was measured. Bitter sensitivity was found to be significantly correlated to the intake of the sweet sea-buckthorn and lingonberry juices; the most bitter-sensitive children exhibited the highest intake of these juices. The opposite relationship was found for bitter sensitivity and the intake of the bitter grapefruit juice. Sour, sweet, and salt sensitivities did not affect the intake of any of the juices. Liking scores were not affected by sensitivity. In conclusion, bitter sensitivity appears to influence food intake in children to a greater extent than sour, sweet, or salt sensitivity. Bitter-sensitive children exhibited a reduced intake of grapefruit juice and a higher intake of sucrose-sweetened juices. Thus, bitter sensitivity might be a challenge in the acceptance of certain bitter foods.
\end{abstract}

(c) 2013 Elsevier Ltd. All rights reserved.

\section{Introduction}

A number of factors influence the acceptance of novel foods by children. One of these factors is how we perceive food, and this factor serves as an important factor for dietary behaviour because children eat what they like (Birch, 1979). The perception of food is influenced by individual taste sensitivity and children appear to be more guided by their taste sensitivity than adults (Mennella, Pepino, \& Reed, 2005). In contrast, adults are more easily able to overcome these genetic influences due to several years of experience.

Bitterness has been widely investigated because humans have an innate aversion to bitter tastes (Steiner, 1974) and because bitterness often constitutes a barrier to healthy eating. The ability of humans to detect extremely low concentrations of bitterness serves as an important evolutionary adaptation for limiting or avoiding the consumption of potentially toxic plant foods

\footnotetext{
Acknowledgements: We thank all the schools, teachers, and children who participated in the study. This study forms part of the OPUS ("Optimal well-being, development and health for Danish children through a healthy New Nordic Diet") project and is supported by a grant from the Nordea Foundation.

* Corresponding author.

E-mail address: dlhh@food.ku.dk (D. Hartvig).
}

(Rozin, 1976). However, in the modern world, bitter taste may impact nutrition and health because many healthy foods, such as fruits and vegetables, contain bitter components. A large number of structurally diverse bitter compounds, including several classes of polyphenols, exist. Polyphenols provide positive and important health benefits by acting as antibacterial and antioxidant agents (D'archivio et al., 2007) and therefore positively impact the human diet.

Large individual differences in bitter perception are a known characteristic in humans (Falconer, 1947; Fischer \& Griffin, 1963; Kalmus, 1971). Genetically predisposed differences in bitter taste sensitivity to 6-n-propylthiouracil (PROP) have been investigated in several studies (e.g., Drewnowski, Henderson, \& Shore, 1997; Fisher et al., 2012). The bitter sensitivity to the bitter component quinine has been rarely measured. The individual variations in the perception of bitter taste are due to allelic diversity in the TAS2R G-protein coupled receptors. To date, 25 bitter receptors have been discovered (Chandrashekar et al., 2000). Base sequence variations in TAS2R19 have been associated with individual differences in the perception of the bitter taste of quinine (Reed et al., 2010) and with differences in the bitterness perception and liking of grapefruit juice (Hayes et al., 2011).

A number of previous studies have investigated the relationship between taste sensitivity and dietary behaviour, and many of these 
studies have attempted to link detection thresholds, identification thresholds, and/or perceived intensity of taste qualities to preferences/liking of foods. Because food contains many structurally diverse taste components, the measurement of only one taste component has a limited ability to predict the ratings of complex foods. However, some previous studies have indicated interesting partial relationships, and others have found no correlations. Fischer, Griffin, England, and Gran (1961) were the first to characterise differences in food preferences by examining quinine sensitivity. These researchers reported that individuals with low thresholds (high sensitivity) for quinine had more food dislikes and aversions than individuals with high thresholds (low sensitivity). Furthermore, quinine has been reported to be a good partial predictor of sucrose intake, i.e., a high quinine intensity rating is associated with high preference and intake of sucrose (Duffy, Peterson, Dinehart, \& Bartoshuk, 2003) and the liking of added sugar (Hayes \& Duffy, 2008). The effect of taste sensitivity to sweet, sour, and salty tastes on food preference and intake has been less investigated. Mattes (1985) found a slight correlation between sweet sensitivity and the liking or intake of sweet-tasting products. Other researchers found no relationship between sucrose perception and the preference for sucrose in adolescents (Coldwell, Oswald, \& Reed, 2009). In addition, a general inverse relationship has been found between the perceived intensities of sourness and the liking or preference of sour-tasting foods in children and adults (Chauhan \& Hawrysh, 1988; Kildegaard, Tønning, \& Thybo, 2011). Studies on the influence of differences in salt taste sensitivity on food acceptance in school-aged children are not prevalent.

Significant correlations in intensity ratings between four stimuli (quinine, citric acid, sucrose, and $\mathrm{NaCl}$ ) have been found ( $\mathrm{Lim}, \mathrm{Ur}$ ban, \& Green, 2008). Additional research on how sensitivity to these taste components is related and whether taste sensitivity affects food intake and liking in children would be valuable.

Familiarity serves as an important factor in children's acceptance of food because it has been found that children prefer foods that they are familiar with. Familiarity accounted for approximately $30 \%$ of the variance in children's preferences for fruits, which is a percentage similar to that obtained for sweetness (Birch, 1979). Therefore, it is important to consider familiarity when measuring acceptance in children.

The New Nordic diet (NND) has been defined in the OPUS project titled "Optimal well-being, development, and health of school children through a New Nordic diet". The concept of the NND is to include more locally produced health-beneficial products e.g., berries and vegetables, into the diet (Mithril et al., 2012). Some of these berries and vegetables are more dominant in bitter and sour tastes than what the children are used to. Because bitter taste is the primary reason why people, particularly children, avoid vegetables and fruits (Rozin \& Vollmerck, 1986), the novelty and sensory properties of the new Nordic foods are likely to be the major barriers for the introduction of these NND components into the children's diet.

This study investigates whether children's taste sensitivity to quinine, citric acid, sucrose, and $\mathrm{NaCl}$ affects their acceptance of novel fruit juices, primarily those made from berries and vegetables that are characteristically found in the Nordic countries. Furthermore, we want to investigate whether sensitivity to these four taste components is related. Our first hypothesis is that taste sensitivity partly influences acceptance of these juices. We expect bitter sensitivity to be oppositely correlated to the acceptance of bitter- and sour-tasting juices and that bitter-sensitive children will exhibit the highest intake of juices that are dominantly sweet. Our second hypothesis is that taste sensitivity to quinine, citric acid, sucrose, and $\mathrm{NaCl}$ is related. To test these hypotheses, taste threshold tests were performed for quinine, citric acid, sucrose, and $\mathrm{NaCl}$. The acceptance of six novel fruit juices, which are characterised as dominant in bitter, sour, or sweet taste, was subsequently measured in 9- to 11-year-old children based on intake and reported liking.

\section{Methods}

\section{Experimental design}

The study was conducted in two different parts: a taste sensitivity test and an acceptance test. The taste sensitivity test was performed twice (with an interval of approximately 3 days) on the four basic tastes: bitter, sour, sweet, and salty. During the acceptance test, all of the participating children evaluated their acceptance of six juices (two per day over a course of three days, see Fig. 1).

\section{Participants}

A total of 328 school children aged 9-11 years participated in the study. They were recruited from five schools in the outskirts of Copenhagen, Denmark. Written parental consent was obtained for all of the participating children. The data were collected during winter 2011/2012. The study was approved by the Research Ethics Committee of Copenhagen (H-1-2010-023).

\section{Taste sensitivity solutions}

The participants were presented with six different concentrations of bitter (quinine hydrochloride, Sigma-Aldrich, Germany) solutions and five concentrations of sour (citric acid, Sigma-Aldrich, Germany), sweet (sucrose, Sigma-Aldrich, Germany), and salty (sodium chloride, Sigma-Aldrich, Germany) solutions. The concentrations of the different tasting solutions are shown in Table 1.

The concentrations of the test solutions were selected based on data from a large study on taste sensitivity performed with 8900 Danish school children (Allesen-Holm, Frøst, \& Bredie, 2009), the International ISO standard concentrations used for the sensory test panel (ISO 3972, 1991E), and pilot test results from a study performed on taste sensitivity in 86 school children (unpublished data).

All of the samples were prepared the day prior to testing, stored at $4{ }^{\circ} \mathrm{C}$, and brought to room temperature before use. The solutions were served in white plastic cups with a lid (29-ml Solo cups, Saebekompagniet, Denmark). Each test solution was marked with a letter from A to $U$ such that the children could keep track of the samples.
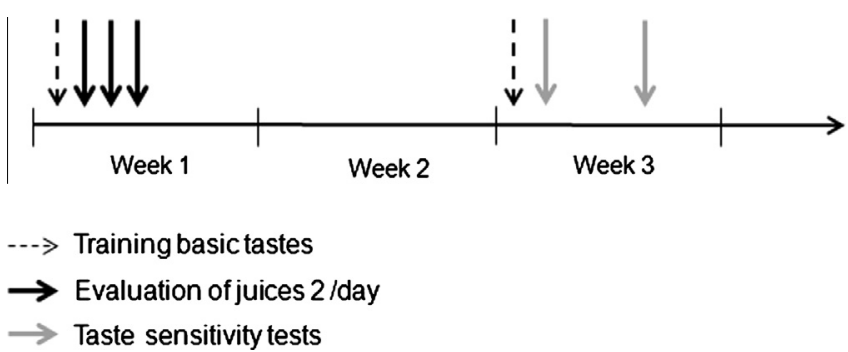

Fig. 1. Experimental design. Two training sessions were performed prior to the sensitivity testing. The acceptance of the juices was evaluated (two juices per day over a three-day period) during week 1 , and the two sensitivity tests were performed during week 3 with an interval of approximately 3 days. 
Table 1

Solutions used in the taste sensitivity test.

\begin{tabular}{|c|c|c|c|c|c|c|c|c|}
\hline \multirow[t]{2}{*}{ Basic taste } & \multirow[t]{2}{*}{ Taste component } & \multicolumn{6}{|c|}{ Concentrations presented (g/L) } & \multirow[t]{2}{*}{ Geometric ratio } \\
\hline & & 1 & 2 & 3 & 4 & 5 & 6 & \\
\hline Bitter & Quinine hydrochloride dihydrate & 0.0014 & 0.0023 & 0.0038 & 0.006 & 0.01 & 0.016 & $R=0.625$ \\
\hline Sour & Citric acid monohydrate & 0.15 & 0.25 & 0.40 & 0.60 & 0.9 & & $R=0.66$ \\
\hline Sweet & Sucrose & 1.5 & 3.0 & 6.0 & 12.0 & 24.0 & & $R=0.5$ \\
\hline Salty & Sodium chloride & 0.35 & 0.55 & 0.85 & 1.3 & 2.0 & & $R=0.65$ \\
\hline
\end{tabular}

All of the tastants were diluted in water. Six bitter solutions and five sour, sweet, and salt solutions were tested.

\section{Experimental procedure taste sensitivity}

Prior to the sensitivity tests, the children attended two training sessions to practice the identification of basic tastes (bitter, sour, sweet, and salty) and the procedure used to taste the solutions. The children were served solutions with medium to high strengths of each of the four basic tastes (quinine $(0.01 \mathrm{~g} / \mathrm{l})$, citric acid $(0.6 \mathrm{~g} /$ $1)$, sucrose $(8.0 \mathrm{~g} / \mathrm{l})$, sodium chloride $(1.3 \mathrm{~g} / \mathrm{l})$ ) and were instructed to taste them one at a time. The tastes of the solutions were discussed in the class, and the basic tastes were related to some products to which the children were familiar with.

Opaque shielding was set up between the children to avoid peer influence during the subsequent sensory testing. The order of samples in the sensitivity tests (bitter, sour, sweet, and salty) was randomised between classes, and the children only had one set of samples on the table at a time. The experiment was commenced with the weakest solutions and progressed in the order of increasing concentration. The children were instructed to rinse their mouth with water between each sample and were provided white opaque cups (36-cl coffee cup, Saebecompagniet, Denmark) to spit in when cleaning their mouth and after tasting the samples. The tasting of the samples was supervised by the project leader to ensure that the children did not rush through the samples and took the needed breaks required to perform the tasting optimally.

For each sample, the children answered the question "Does the sample taste different from water?" with either yes or no. If they marked yes, they were instructed to answer the question "How does it taste?" with the categories "do not know", "sour", "sweet", "salty", or "bitter". Each taste was illustrated with a picture of a food representing the particular taste quality (citrus fruit, white sugar, salt holder, and dark chocolate, respectively) to remind the children of the basic tastes. Similar scales had been used in a previous study with school children (Allesen-Holm et al., 2009). The use of the scales was explained carefully during the training session prior to the first test session and was shortly repeated at the successive test sessions if necessary. It was emphasised that there were no right or wrong answers and that we simply asked for each individual child's opinion.

\section{Experimental foods}

With one exception, the products used in this experiment were composed of Nordic-grown ingredients, i.e., berries and vegetables representing the Nordic tastes that would naturally occur in a Nordic diet. The products used were juices based on one Nordic component dominant in the bitter, sour, or sweet taste. A liquid texture was chosen to enable consumption using a straw and to minimise effects of texture. Furthermore, the products had to be easy to handle and available for ad libitum intake.

The juices used in this experiment were carrot juice (juiced organic carrots; undiluted), rosehip juice (Ekströms kallvispad Nyponsoppa produced by Procordia Food, Sweden; $400 \mathrm{~g}$ in $2.7 \mathrm{~L}$ of water), sea-buckthorn juice (produced by Smartwell, Sweden; containing $10 \%$ sucrose; served undiluted), lingonberry juice (produced by Meyers Foods, Denmark; containing 45\% sucrose; mixed 1:3 with water), grapefruit juice (produced for COOP Denmark; served undiluted), and aronia juice (organic aronia; sour juice; produced by Din Sundhed, Denmark; mixed 1:2 with water). Two of these products were naturally dominant in the sweet taste: carrot juice and rosehip juice. The sea-buckthorn juice and lingonberry juice were naturally sour products even with the presence of the added sucrose. These juices were considered sweetened sour juices. Grapefruit juice and aronia juice were dominant in the bitter taste, and aronia was also very astringent. Grapefruit juice is not a Nordic product but was included because of its bitter component naringin, which was expected to correlate with quinine sensitivity because these compounds activate the same bitter receptor (TAS2R19) (Hayes et al., 2011; Reed et al., 2010).

\section{Acceptance of juices}

The acceptance testing took place in the classrooms preferably in the morning hours (from 9 to 12 a.m.). A few tests were performed in the afternoon. For the testing, the children were seated on their own and not disturbed by the other children. The acceptance testing was conducted over a three-day period, and the children received two products on each test day; the juices were served in randomised pairs and randomised order between classes. The products were prepared in the morning prior to testing and were served at room temperature in opaque cardboard cups (Rioba 0.18-1 coffee cup, Metro Cash \& Carry, Denmark) covered with an opaque lid and containing a black straw to eliminate any visual influences. The children were given the possibility of ad libitum intakes of the products to avoid ceiling effects. Each cup was weighed ( $\pm 1 \mathrm{~g}$ ) before and after serving to measure the total consumption of each product. The children evaluated their liking of each juice by answering the question "How much do you like the product?" on a 7-point hedonic smiley scale ranging from "dislike a lot" to "like a lot" (Chen \& Resurreccion, 1996; Guinard, 2001). To evaluate familiarity, the children were asked "How well do you know this taste?" on a 5-point scale ranging from "not at all" to "very". The children evaluated the taste characteristics of the juices by answering the question "what basic taste do you find in the product?" on a 5-point scale with the possibility of marking "do not know", "sour", "sweet", "salty", or "bitter". They were instructed to mark one taste if possible (maximum of two). To adjust for the influence of hunger/satiety on the intake data, the children were asked to score their appetite on a 5-point bipolar scale ranging from "very satiated" to "very hungry" before each product tasting. The use of the scales and the parameters were explained carefully during a training session prior to the first test session. It was emphasised that there were no right or wrong answers and that we simply asked for each individual child's opinion.

\section{Statistical analysis}

The intake in weight $(\mathrm{g})$ and the children's reported liking of each of the six juices were included as response variables in GLM 
analyses. Furthermore, Pearson's correlation analyses were used to analyse the data.

GLM analyses were performed to test whether taste sensitivity had an effect on the intake and reported liking of the six juices. The intake data were log-transformed to stabilise the variance. The bitter, sour, sweet, and salt sensitivities were included as factor variables in the analyses in an individual basis to avoid covariance. The school, class, and gender were included as fixed factors in all of the analyses. Hunger was included as a covariate in the analyses to correct for the influence of hunger on the response variables. The children's reported familiarity was also included as a covariate. Any interactions were included if relevant. Only significant factors and covariates were included in the final model, and post hoc tests were performed if the factor variables were found to be significant.

Correlations between sensitivity to the bitter, sour, sweet, and salty taste were tested. All analyses were performed in IBM SPSS version 19 . The alpha criterion for significance was $5 \%$.

\section{Results}

Sensitivity grouping of children

The children were subjected to two taste sensitivity tests, and the data of the second test were used for further analysis. The data from the initial taste test was considered to be biased because the children were learning how to perform the test. The data were divided based on the children's identification of the respective basic taste. The grouping based on this identification provided a more balanced division of the children than detection. Because the children performed two training sessions on the basic tastes prior to the sensitivity test, it was assumed that identification was a reliable measure of the taste sensitivity threshold. For bitterness, the children were divided into seven different groups based on the concentration at which they consistently correctly identified the bitter taste. For the sour, sweet, and salty tastes the children were divided into six groups using similar criteria (Table 2). Group 1 contained the most/high-sensitive children and groups 7 (bitter) and 6 (sour, sweet, and salty) contained the least/non-sensitive children. If the answers were too unclear to interpret the level of identification, the data were not included; therefore, the value of $N$ differed between the four taste qualities.

\section{Characteristics of the different sensitivity groups}

The children were divided into groups according to their taste sensitivity for the basic tastes as observed in Table 3. For the bitter taste sensitivity, the lowest number of children were categorised as the most/high-sensitive (group 1; 5.9\%), and the largest groups consisted of the high/medium-sensitive children (groups 2 and 3; $23.7 \%$ and $20.1 \%$, respectively). The rest of the children were evenly distributed in the other groups. For sour taste sensitivity, only a few children were able to identify the two lowest concentrations and thus grouped in the most/high-sensitive groups for sour taste (groups 1 and 2; 2.3\% and 3.4\%, respectively). More than half of the children were categorised in the medium sour-sensitive group (group $4 ; 58.9 \%$ ). For sour, sweet, and salty sensitivity, only a few children were unable to taste the highest solutions (groups 6 and $7 ; 2.6 \%$ and $1.7 \%$, respectively).

The genders were equally represented in the sensitivity groups with the exception of the highly bitter-sensitive children (bitter group 1). Thirteen children were characterised as highly bitter sensitive for quinine: 10 boys and 3 girls.

\section{Correlations between taste stimuli}

The correlations of the four different sensitivity groupings against each other revealed only relatively weak correlations; however, all of these were significant (Table 4). The strongest correlation was observed for sweet and salty $(r=0.35 ; p<0.001)$ sensitivities and the least correlation was obtained for bitter and sour ( $r=0.17 ; p=0.017)$ sensitivities.

\section{Product taste characteristics}

The children were able to evaluate the basic taste properties of the juices, and the mean taste scores for the six juices are shown in

\section{Table 4}

Correlation coefficients $(r)$ between stimuli.

\begin{tabular}{llll}
\hline & Quinine & Citric acid & Sucrose \\
\hline Citric acid & $0.17, P=0.017$ & & \\
Sucrose & $0.29, P<0.001$ & $0.31, P<0.001$ & \\
$\mathrm{NaCl}$ & $0.30, P<0.001$ & $0.33, P<0.001$ & $0.35, P<0.001$ \\
\hline
\end{tabular}

Table 2

Grouping of children based on taste sensitivity.

\begin{tabular}{|c|c|c|c|c|c|c|c|}
\hline Group & 1 & 2 & 3 & 4 & 5 & 6 & 7 \\
\hline Bitter & $\begin{array}{l}\text { Identified all of } \\
\text { the samples }\end{array}$ & $\begin{array}{l}\text { Identified } \\
\text { samples 2-6 }\end{array}$ & $\begin{array}{l}\text { Identified } \\
\text { sample 3-6 }\end{array}$ & $\begin{array}{l}\text { Identified } \\
\text { samples 4-6 }\end{array}$ & $\begin{array}{l}\text { Identified } \\
\text { samples 5-6 }\end{array}$ & Identified sample 6 & $\begin{array}{l}\text { Did not identify a taste in } \\
\text { any of the samples }\end{array}$ \\
\hline $\begin{array}{l}\text { Sour, sweet, } \\
\text { and salty }\end{array}$ & $\begin{array}{l}\text { Identified all of } \\
\text { the samples }\end{array}$ & $\begin{array}{l}\text { Identifies } \\
\text { samples 2-5 }\end{array}$ & $\begin{array}{l}\text { Identified } \\
\text { samples 3-5 }\end{array}$ & $\begin{array}{l}\text { Identified } \\
\text { samples } 4-5\end{array}$ & $\begin{array}{l}\text { Identified } \\
\text { sample } 5\end{array}$ & $\begin{array}{l}\text { Did not identify a taste in } \\
\text { any of the samples }\end{array}$ & \\
\hline
\end{tabular}

Group 1: most/high-sensitive children. Groups 7 (bitter) and 6 (sour, sweet, and salt): least/non-sensitive children.

Table 3

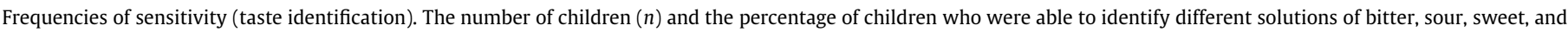
salt tastants in the second sensitivity test are shown.

\begin{tabular}{|c|c|c|c|c|}
\hline Sensitivity group (identification) & Bitter $n$ (percent) & Sour $n$ (percent) & Sweet $n$ (percent) & Salt $n$ (percent) \\
\hline 1 (most sensitive) & $13(5.9)$ & $6(2.3)$ & $27(10.2)$ & $61(26.4)$ \\
\hline 2 & $52(23.7)$ & $9(3.4)$ & $91(34.5)$ & $37(16.0)$ \\
\hline 3 & $44(20.1)$ & $26(9.8)$ & $69(26.1)$ & $44(19.0)$ \\
\hline 4 & $32(14.6)$ & $156(58.9)$ & $37(14.0)$ & $41(17.7)$ \\
\hline 5 & $30(13.7)$ & $61(23.0)$ & $37(14.0)$ & $44(19.0)$ \\
\hline 6 & $22(10.0)$ & $7(2.6)$ & $3(1.1)$ & $4(1.7)$ \\
\hline 7 (least sensitive) & $26(11.9)$ & & & \\
\hline Total $n$ (percent) & $219(100)$ & $265(100)$ & $264(100)$ & $231(100)$ \\
\hline
\end{tabular}




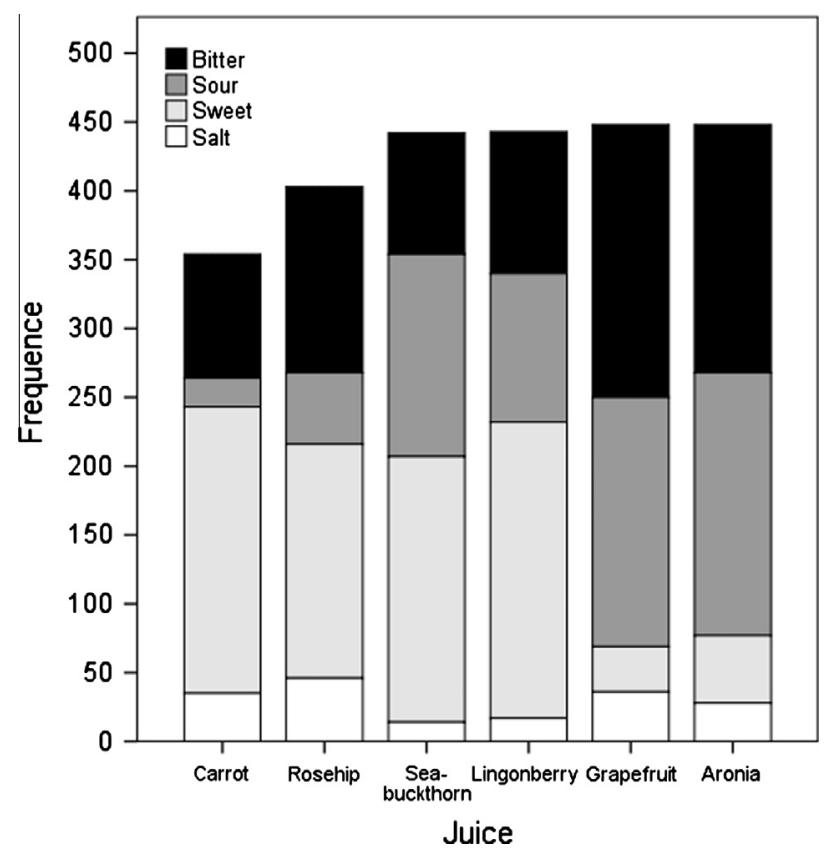

Fig. 2. Taste characteristics of the six Nordic juices. The frequency plot is based on the children's own taste evaluation of the products.

Fig. 2. The rosehip juice and carrot juice were intended to represent sweet products and were characterised as such by the participants. The rosehip juice was also characterised as slightly little bitter. The children characterised the sea-buckthorn juice as primarily sweet and less although still sour. The children judged the lingonberry juice as primarily sweet and characterised the grapefruit juice as dominant in bitter taste and to a lesser degree in sour taste. The aronia juice was characterised as bitter and sour.

\section{Acceptance of juices in relation to taste sensitivity}

The results of the GLM analyses performed on the relationship between acceptance and taste sensitivity grouping of the children are shown in Table 5. The results are divided into the sensitivity for each of the basic tastes.

\section{Bitter sensitivity and juice acceptance}

The sensitivity of the children for bitter taste was significantly related to the intake of sea-buckthorn juice $(p=0.041)$. The highly bitter-sensitive children (group 1) had the highest intake, and the least/non-sensitive children had the lowest intake of sea-buckthorn (Fig. 3). A similar relationship was observed for the intake of lingonberry juice ( $p=0.033$; Fig. 4 ). The bitter taste sensitivity also influenced the intake of grapefruit juice $(p=0.012)$ : the least-sensitive groups of children (groups 6-7) had the highest

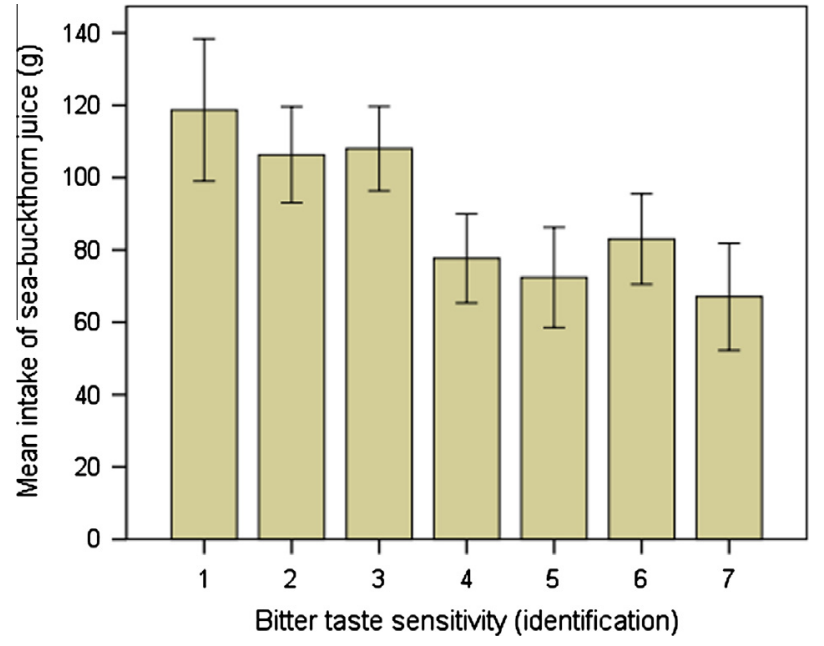

Fig. 3. Mean intake $( \pm S E M)$ of sea-buckthorn juice in relation to bitter taste sensitivity (identification) of groups 1-7: group $1(n=13$; most-bitter-sensitive children), group $2(n=52)$, group $3(n=44)$, group $4(n=32)$, group $5(n=30)$, group $6(n=22)$, and group 7 ( $n=26$; least/non-bitter-sensitive children).

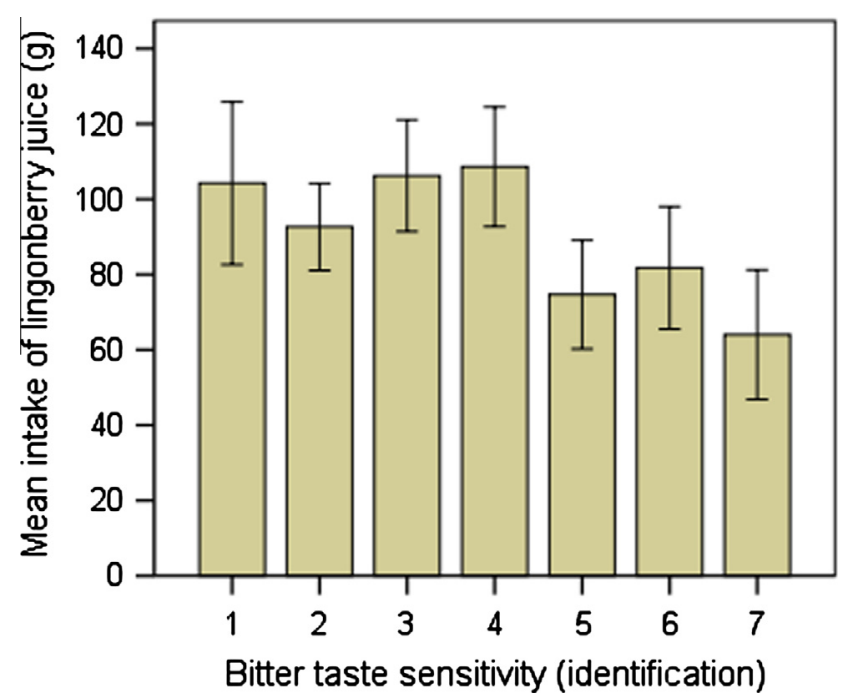

Fig. 4. Mean intake ( \pm SEM) of lingonberry juice in relation to bitter taste sensitivity (identification) of groups 1-7: group 1 ( $n=13$; most-bitter-sensitive children), group $2(n=52)$, group $3(n=44)$, group $4(n=32)$, group $5(n=30)$, group 6 $(n=22)$, and group 7 ( $n=26$; least/non-bitter-sensitive children).

intake, and the medium and most bitter-sensitive children had the lowest intake of grapefruit juice (Fig. 5). The intake of carrot, rosehip, and aronia juices was not affected significantly by the bitter sensitivity of the children (Table 5). There was no effect of gender, whereas familiarity had a significant effect on the intake of

Table 5

Results of the GLM tests comparing the taste sensitivity with the intake of the six juices. The gender, class, familiarity, and hunger variables were included in the model.

\begin{tabular}{|c|c|c|c|c|}
\hline Product & Bitter sensitivity & Sour sensitivity & Sweet sensitivity & Salt sensitivity \\
\hline Carrot & NS & NS & NS & NS \\
\hline Rosehip & NS & NS & NS & NS \\
\hline Sea-buckthorn & $P=0.041 \downarrow$ & NS & NS & NS \\
\hline Lingonberry & $P=0.033 \downarrow$ & NS & NS & NS \\
\hline Grapefruit & $P=0.012 \uparrow$ & NS & NS & NS \\
\hline Aronia & NS & NS & NS & NS \\
\hline
\end{tabular}

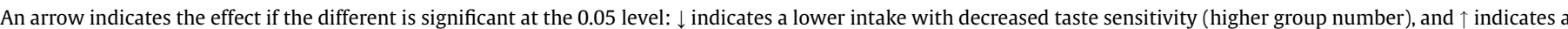
higher intake with decreased taste sensitivity (higher group number). 


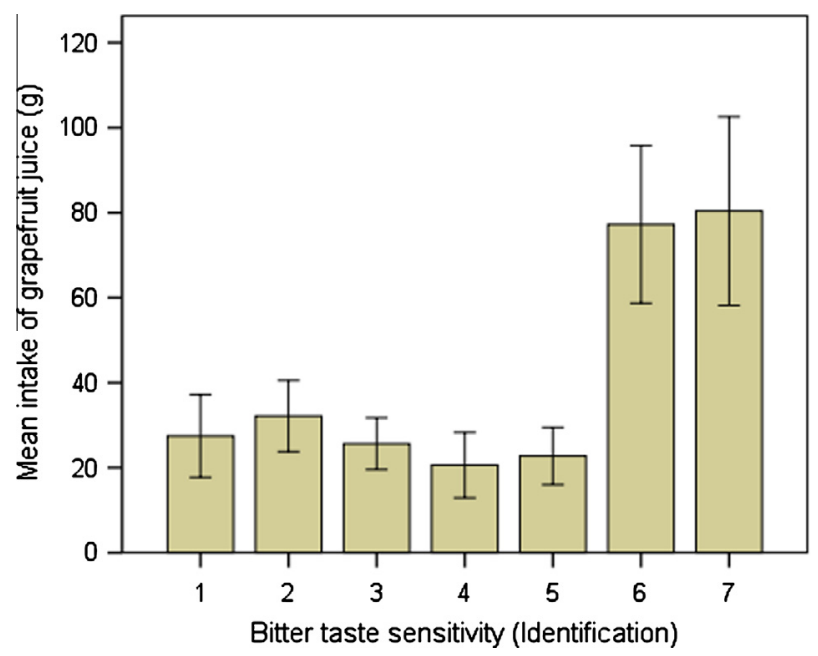

Fig. 5. Mean intake ( \pm SEM) of grapefruit juice in relation to bitter taste sensitivity (identification) of groups 1-7: group $1(n=13$; most-bitter-sensitive children), group $2(n=52)$, group $3(n=44)$, group $4(n=32)$, group $5(n=30)$, group 6 ( $n=22)$, and group 7 ( $n=26$; least/non-bitter-sensitive children).

rosehip $\quad(p=0.002)$, sea-buckthorn $\quad(p=0.001)$, grapefruit $(p=0.001)$, and aronia juices $(p=0.000)$. The reported liking of any of the juices was not significantly affected by the bitter sensitivity. This result indicated that bitterness is more related to juice intake than to the overall liking response for the juices.

\section{Sour, sweet, and salt sensitivities and juice acceptance}

The sour, sweet, and salty taste sensitivities did not significantly affect the intake of the juices. Familiarity had a significant effect in the intake of the juices if sour sensitivity was included in the model: $\operatorname{carrot}(p=0.004)$, rosehip $(p=0.000)$, sea-buckthorn $(p=0.031)$, lingonberry $(p=0.000)$, grapefruit $(p=0.000)$, and aronia $(p=0.000)$. When the analysis was performed with the inclusion of sweet sensitivity in the model, familiarity was found to have a significant effect on the intake of rosehip $(p=0.000)$, lingonberry $(p=0.002)$, grapefruit $(p=0.000)$, and aronia juices $(p=0.000)$. When the analyses included salt sensitivity in the model, familiarity was found to exert a significant impact on the intake of carrot $(p=0.032)$, rosehip $(p=0.002)$, grapefruit $(p=0.003)$, and aronia juices $(p=0.018)$.

The product liking was not significantly affected by sour, sweet, or salt sensitivity. However, familiarity was found to be significant in all of the analyses performed.

\section{Discussion}

\section{Correlation between taste sensitivities for basic tastes}

Positive but relatively weak correlations were found in this study between sensitivity to quinine, citric acid, sucrose, and $\mathrm{NaCl}$ tastes. Previous findings by Lim et al. (2008) showed significant correlated intensity ratings between quinine, sucrose, citric acid, and $\mathrm{NaCl}$ solutions. A positive relationship between quinine and sucrose detection thresholds has been previously reported (Chang, Chung, Kim, Chung, \& Kho, 2006). As indicated by several studies, it seems reasonable that subjects that are highly sensitive to bitter taste have a high sensitivity for other sensory attributes, such as other tastes and textures.
Gender difference in sensitivity

Our finding that children with different genders were similarly divided in the different sensitivity groups for sweet and bitter tastes is in accordance with the findings reported by Chang et al. (2006). These researchers reported no gender differences in taste detection and recognition for sucrose and quinine. However, we observed that a higher number of boys are highly sensitive to quinine. This result is considered a coincidence because all other groups exhibit an equal number of female and male children. We also found gender to be equally distributed in the taste sensitivity groups obtained for the sour and salty tastes.

\section{Scale and solutions used for sensitivity testing}

This sensitivity method was successfully used in a previous study by Allesen-Holm et al. (2009), who studied 8900 5- to 20 -year-old individuals, of which approximately $60 \%$ were 9 - to 11-year-old children. Furthermore, this method has been used in a pilot study that was conducted with 9- to 11-year-old children $(n=86)$. In the present study, the scale was successfully used, and it appeared that the children found it easy to understand. Furthermore, there were helpers present to guide the children if they were unsure how to use the scale. If the children were confused about the scale, this confusion was often revealed in their results because they only had to answer the question on basic tastes if the sample tasted differently from water.

The concentrations used for citric acid and sucrose were inspired by the results of various studies, including that conducted by Allesen-Holm et al. (2009). There researchers used $0.6 \mathrm{~g} / \mathrm{l}$ as the highest concentration citric acid concentration and found that approximately $30 \%$ of the participants were not able to identify the sour taste; therefore, we included a solution with a higher concentration of citric acid $(0.9 \mathrm{~g} / \mathrm{l})$. Unfortunately, the results from the present study revealed an unbalanced division of children based on sour taste; most children identified sour taste with the fourth-highest concentration. This unbalance in identification was not found to the same extent in either of the previous studies that used similar solutions. This different may be ascribed an insufficient interval for citric acid concentrations in this age group. This unbalanced division of children is a problem in the analyses because some of the sour sensitivity groups are represented by very few children, which might have influenced the results for sour sensitivity. In future studies on the identification of sour sensitivity by 9- to 11-year-old children, the use of citric acid concentrations should be adjusted because approximately half of the children were found to identify citric acid at a concentration of approximately $0.6 \mathrm{~g} / \mathrm{l}$.

\section{Identification and characterisation of basic tastes by children}

To reduce the occurrence of confusion between the four basic tastes (bitter, sour, sweet, and salty) used in the sensitivity test, two training sessions on basic tastes were performed prior to the first sensitivity test. The purposes of the training sessions were to make the children more aware of and familiar with the basic tastes. The basic tastes were discussed and linked to different products that the children usually eat. Although it is not possible to completely avoid confusion, we believe that the two training sessions on basic tastes and one test session with the test solutions were sufficient training to conduct a reliable sensitivity test. Less clear responses, i.e., responses that did not imply a clear identification level, were excluded. The responses for bitter taste were more frequently excluded than those for the other basic tastes. Therefore, the total numbers of responses included in the data analyses were different for the different basic tastes. The confusion 
regarding bitter taste may in part explain why the bitter responses were most frequently removed from the analysis. Astringency was not included as a possible characteristic of juices because the emphasis was on basic tastes. Furthermore, it was assumed that a question of astringency would be too complex for the children. Some of the bitter responses in the characterisation may have been due to other oral irritations from either acids or astringency, because the juices were more complex than simply bitter, sour, or sweet.

\section{Acceptance of the products in relation to taste sensitivity}

Only few studies have investigated the taste sensitivity to quinine, citric acid, sucrose, and $\mathrm{NaCl}$ and its relationship to food acceptance or preference in children. Most research studies have been performed on adults, and quite different methods have been used, which makes a comparison of the results difficult. Furthermore, the products used for the investigation of food acceptance or preferences are complex mixtures of many structurally diverse taste substances. Therefore, the measurement of sensitivity for few taste substances has a limited ability to predict the acceptance or preference of specific products; however, several studies have reported partial associations.

\section{Quinine sensitivity}

The hypothesis that the acceptance of bitter fruit juice is related to quinine taste sensitivity in 9- to 11-year-old children was found to be partly true. The intake of the bitter grapefruit juice was related to the sensitivity for quinine: the highly sensitive and medium-sensitive children exhibited a lower intake of grapefruit juice than the less/non-sensitive children. Drewnowski et al. (1997) reported a similar relationship between PROP sensitivity and hedonic evaluation of grapefruit juice in adults. However, a relationship between quinine taste sensitivity and grapefruit juice has not been reported previously. Individual variations in the perception of bitter taste components are a common human trait (Falconer, 1947; Fischer \& Griffin, 1963; Kalmus, 1971) that reflects the allelic diversity in TAS2R receptors. Sequence variations in TAS2R19 have been associated with individual differences in the bitter taste of quinine (Reed et al., 2010). In addition, sequence variations in TAS2R19 appear to be partially responsible for the differences in the perception of grapefruit juice (Hayes et al., 2011). Because the perceptions of quinine and grapefruit juice are associated with sequence variations in TAS2R19, the relationship found in this study between quinine sensitivity and grapefruit intake appears reasonable. In the present study, the intake and liking of aronia was not found to be related to quinine bitter sensitivity. This was an unexpected finding because aronia was perceived as a bitter and sour product. The reason for this result could be that the bitter components of aronia activate different bitter receptors than those activated by quinine. Another reason could be the high astringency of aronia (Chrubasik, Li, \& Chrubasik, 2010). The sensation of astringency on the human palate has been defined as a complex group of sensations involving dryness of the oral surface and tightening and puckering sensations of the mucosa and muscles around the mouth (Lee \& Lawless, 1991). Individual differences in the astringency response are not related to differences in the perceived responses to bitterness or sourness (Dinella, Recchia, Tuorila, \& Monteleone, 2011). It is possible that the astringency of aronia dominated the oral sensation and that therefore the acceptance of aronia was related to the individual astringency responses and not quinine sensitivity. Similarly to bitter taste, astringency has a negative effect on acceptance (Jaeger, Axten, \& Wohlers, 2009).
The acceptance of sea-buckthorn and lingonberry juices, which were both characterised by the children as products that are dominantly sweet, was found to be significantly related to quinine sensitivity in this study. The highly bitter-sensitive children had a significantly higher intake of both of these juices than the non/ less-bitter-sensitive group of children. However, the acceptance of carrot and rosehip juices was not related to bitter sensitivity, even though these juices were dominant in the sweet taste. This may be because these are naturally sweet and do not contain added sucrose, i.e., sucrose may be closely related to quinine sensitivity. According to Hayes and Duffy (2008), high sensitivity to quinine appears to be a good predictor of high sucrose preference in adults. Subjects who tasted $0.32 \mathrm{mM}$ quinine as more bitter than $3.2 \mathrm{mM}$ PROP needed more sucrose to reach hedonic optima compared with subjects who found PROP to be more bitter than quinine. Furthermore, Duffy et al. (2003) investigated a relationship between high quinine intensity ratings and high preference and intake of sucrose. The reason why bitter-sensitive children favour sweet foods is unclear. Possibly these children perceive many other foods to be too bitter or strong tasting and therefore eat more sweet foods to compensate for the bitter foods they avoid. In addition, the sweetness might simply mask the bitterness of products and therefore makes bitter-tasting foods less unpleasant to bitter-sensitive children.

\section{Sour, sweet, and salt taste sensitivities}

The intakes of the juices were not significantly affected by the sensitivities for sour, sweet, and salty tastes. Few studies have analysed the relationship between the sensitivities for sour, sweet, and salty tastes and food acceptance, and this study did not find any such relationships for sour, sweet, and salty tastes.

A general inverse relationship has been found between the perceived intensities of sourness in products and the liking or preference in children and adults (Chauhan \& Hawrysh, 1988; Kildegaard et al., 2011). However, Liem and Mennella (2003) found that approximately one third of the 5- to 9-year-old children studied appeared to prefer the extremely sour taste. Their preference increased with increasing levels of citric acid. Based on their perceived sourness in a multiple ranking test with different citric acid concentrations, the authors assumed that the extreme preference for sour taste in this group of children could not be assigned a lower capability to perceived sourness. The complex food matrix used in the study was gelatins with a sweet background taste due to added sucrose and glucose. The sea-buckthorn juice used in the present study also tasted both sweet and sour, and many children liked this product. Despite of the sourness of sea-buckthorn juice, its intake was not related to sour taste sensitivity.

Mattes (1985) found a slight correlation between sweet sensitivity and liking and intake of sweet-tasting products. Another study found that the perceived intensity of sweetness was not significantly related to liking, frequent intake, and cravings for sweet foods in adults (Keskitalo et al., 2007). If a clear relationship between sweet taste sensitivity and acceptance of sweet foods exists in children, we would have expected correlations between the measured sweet taste sensitivity and the intake of juices with added sucrose (sea-buckthorn and lingonberry) because sucrose was used in the taste sensitivity test. Sensitivity to a particular sweet substance (e.g., sucrose) does not necessary predict sensitivity to other sweet substances (Faurion, 1987).

Salty sensitivity varies between different individuals. Mitchell, Brunton, and Wilkinson (2013) found no associations between individual recognition thresholds of salty-tasting solutions and the acceptance of regular- and reduced-sodium vegetable soups in adults. Studies on the influence of differences in salt taste sensitivity on food acceptance in school-aged children are not prevalent. 
We did not include salty products in the present study, but our results do not indicate any significant relationships between salty taste sensitivity and the acceptance of juices dominant in the bitter, sour, and sweet tastes in children.

\section{Familiarity}

Familiarity was significant in most analyses on intake and all analyses on liking. This finding was not surprising because familiarity constitutes a very important factor in the food choice of children: children tend to prefer familiar products compared with less familiar products (Birch, 1979). In the present study, some of the products used, especially the berry products, such as sea-buckthorn and aronia, were unfamiliar to the children, whereas others were, such as carrot, familiar to most children. This study stresses the importance of evaluating familiarity when measuring intake and liking in children.

\section{Acceptance measure}

The acceptance of the juices was measured in terms of intake and reported liking. Significant differences were found in the intake but not in the liking scores. This result could be due to the differences in taste sensitivity being more reflected in the actual intake than the liking of a product. Bitter-sensitive children do not necessarily have a higher liking for sweetness than the less-bitter-sensitive children. Another possible explanation for the differences between the intake and the liking results could be that the intake range was wider compared to the 7-point scale used to measure liking. Children tend to avoid the lower (negative) end of the hedonic scale (Moskowitz, 1994), which may cause a narrow spread in the reported liking. However, a similar 7-point hedonic scale has been successfully used elsewhere, from the age of 5 years (Chen \& Resurreccion, 1996). Several studies that investigated children's acceptance of food have reported intake as a reliable direct measure of acceptance (Dinehart, Hayes, Bartoshuk, Lanier, \& Duffy, 2006; Fisher et al., 2012; Hausner, Hartvig, Reinbach, Wendin, \& Bredie, 2012).

\section{Acceptance of the New Nordic products}

With the exception of grapefruit, the juices used in this study represent typical Nordic berries and vegetables as components of the New Nordic Diet.

Because sweetness is an important dimension that determines children's food preferences (Birch, 1979), it will be a challenge to induce children to accept some of the components of the New Nordic Diet, which are more bitter and/or less sweet than the foods that children usually eat in Denmark. This is particularly true for bitter-sensitive children. The high acceptance of sea-buckthorn and lingonberry juices by bitter-sensitive children in this study is most likely due to the addition of sucrose and might not have occurred without the addition of sucrose, because sweet and bitter tastes inhibit one another (Keast \& Breslin, 2002). In a study on sea-buckthorn sweetness had strong positive effects on juice pleasantness and adding $6.5 \%$ of sucrose increased pleasantness markedly (Tang, Kälviäinen, \& Tuorila, 2001). The natural form of seabuckthorn has a unique strong fruity flavour and a sour taste, but it has also been characterised by astringency and bitterness (Tang et al., 2001; Tiitinen, Hakala, \& Kallio, 2005). Lingonberry is a sour/ bitter juice with an astringent aftertaste. From a health perspective, it is not sensible to add sucrose to naturally bitter- and sour-tasting products to increase their acceptance by bitter-sensitive children. To make a healthy juice that would be highly accepted by bitter-sensitive children, a solution might be to mix the bitter/sour berries/fruits or vegetables with naturally sweet berries/fruits or other components to mask the bitterness. Kildegaard et al. (2011) found a decrease in the perceived sourness of juices in 9- to 14-year-old children when the dry matter was increased; therefore, the adjustment of the dry content of juices may also be an alternate approach to alter the sourness of juices. A study on berries (Sea-buckthorn, lingonberry, bilberry, blackcurrent) indicated that children prefer berries in a matrix like yoghurt, which is apparently capable of rounding of the sour taste caused by organic fruit acids (Suomela et al., 2012). In a study by Fisher et al. (2012), the intake of raw broccoli in PROP-sensitive children was successfully increased by $80 \%$ when the broccoli was provided with dressing. Due to differences in bitter-taste sensitivity, more diverse and complemented food offerings may be needed to make healthy components more palatable for all children.

\section{Conclusions}

This study supports earlier findings that link taste perception with dietary behaviour, specifically with regard to the sensitivity to bitter taste (quinine) in children. The present results indicate that quinine sensitivity is more reflected by the intake than the liking of products. The highly bitter-sensitive children had a lower intake of bitter grapefruit juice and an increased intake of juices with added sucrose, namely sea-buckthorn and lingonberry juice, compared to the less/non-bitter-sensitive children. This finding might reflect the challenge of encouraging the NND, which contains more naturally bitter products and less sugar, especially in highly bittersensitive children. This group of children may need to be handled slightly differently and be provided diversified offerings when introduced to some of the new Nordic food components. Furthermore, the present study indicates significant, however weak, correlations among the taste sensitivity measures for quinine, citric acid, sucrose, and $\mathrm{NaCl}$.

\section{References}

Allesen-Holm, B. H., Frøst, M. B. \& Bredie, W. L. P. (2009). Taste sensitivity and preferences in Danish school children. In Proceedings of the 8th Rose Marie Pangborn Symposium, 26-30 July, Florence, Italy.

Birch, L. L. (1979). Dimensions of preschool children's food preferences. Journal of Nutrition Education, 11, 77-80.

Chandrashekar, J., Mueller, K. L., Hoon, M. A., Adler, E., Feng, L. X., Guo, W., et al (2000). T2Rs function as bitter taste receptors. Cell, 100, 703-711.

Chang, W. I., Chung, J. W., Kim, Y. K., Chung, S. C., \& Kho, H. S. (2006). The relationship between phenylthiocarbamide (PTC) and 6-n-propylthiouracil (PROP) taster status and taste thresholds for sucrose and quinine. Archives of Oral Biology, 51, 427-432.

Chauhan, J., \& Hawrysh, Z. J. (1988). Suprathreshold sour taste intensity and pleasantness perception with age. Physiology \& Behavior, 43, 601-607.

Chen, A. W., \& Resurreccion, A. V. A. (1996). Age appropriate hedonic scales to measure food preferences of young children. Journal of Sensory Studies, 11, 141-163.

Chrubasik, C., Li, G., \& Chrubasik, S. (2010). The clinical effectiveness of chokeberry. A systematic review. Phytotheraphy Research, 24, 1107-1114.

Coldwell, S. E., Oswald, T. K., \& Reed, D. R. (2009). A marker of growth differs between adolescents with high vs. low sugar preference. Physiology E Behavior, 96, 574-580.

D’archivio, M., Filesi, C., Benedetto, R. D., Gargiulo, R., Giovannini, C., \& Masella, R. (2007). Polyphenols, dietary sources and bioavailability. Ann Ist Super Sanita (Annali dell'Istituto Superiore di Sanita), 43, 348-361.

Dinehart, M. E., Hayes, J. E., Bartoshuk, L. M., Lanier, S. L., \& Duffy, V. B. (2006). Bitter taste markers explain variability in vegetable sweetness, bitterness, and intake. Physiology \& Behavior, 87, 304-313.

Dinella, C., Recchia, A., Tuorila, H., \& Monteleone, E. (2011). Individual astringency responsiveness affects the acceptance of phenol-rich foods. Appetite, 56, 633-642.

Drewnowski, A., Henderson, S. A., \& Shore, A. B. (1997). Taste responses to naringin, a flavonoid, and the acceptance of grapefruit juice are related to genetic sensitivity to 6-n-propylthiouracil. American Journal of Clinical Nutrition, 66, 391-397.

Duffy, V. B., Peterson, J. M., Dinehart, M. E., \& Bartoshuk, L. M. (2003). Genetic and environmental variation in taste, associations with sweet intensity, preference, and intake. Clinical Nutrition, 18, 209-220.

Falconer, D. S. (1947). Sensory thresholds for solutions of phenyl-thio-carbamide. Annals of Eugenics, 13, 211-222. 
Faurion, A. (1987). Physiology of the sweet taste. In H. Autrum, D. Ottoson, E. Perl, R. Schmidt, H. Shimazu, \& W. Willis (Eds.), Progress in sensory physiology. Berlin: Springer-Verlag.

Fischer, R., \& Griffin, F. (1963). Quinine dimorphism. A cardinal determinant of taste sensitivity. Nature, 200, 343-347.

Fischer, R., Griffin, F., England, S., \& Gran, S. M. (1961). Taste thresholds and food dislikes. Nature, 191, 1328.

Fisher, J. O., Mennella, J. A., Hughes, S. O., Liu, Y., Mendoza, P. M., \& Patrick, H. (2012). Offering "dip" promotes intake of a moderately liked raw vegetable among preschoolers with genetic sensitivity to bitterness. Journal of the Academy of Nutrition and Dietetics, 112, 235-245.

Guinard, J. X. (2001). Sensory and consumer testing with children. Trends in Food Science \& Technology, 11, 273-283.

Hausner, H., Hartvig, D. L., Reinbach, H. C., Wendin, K., \& Bredie, W. L. P. (2012). Effects of repeated exposure on acceptance of initially disliked and liked Nordic snack bars in 9-11-year-old children. Clinical Nutrition, 31, 137-143.

Hayes, J. E., \& Duffy, V. B. (2008). Oral sensory phenotype identifies level of sugar and fat required for maximal liking. Physiology \& Behavior, 95, 77-87.

Hayes, J. E., Wallace, M. R., Knopik, V. S., Herbstman, D. M., Bartoshuk, L. M., \& Duffy, V. B. (2011). Allelic variation in TAS2R bitter receptor genes associates with variation in sensations from and ingestive behaviors toward common bitter beverages in adults. Chemical Senses, 36, 311-319.

Jaeger, S. E., Axten, L. G., \& Wohlers, M. W. (2009). Sun-waterhouse. Polyphenol-rich beverages. Insights from sensory and consumer science. Journal of the Science of Food and Agriculture, 89, 2356-2363.

Kalmus, H. (1971). Genetics of taste. Handbook of sensory physiology. Berlin: Springer Beidler LM.

Keast, S. J. R., \& Breslin, P. A. S. (2002). An overview of binary taste-taste interactions. Food Quality and Preference, 14, 111-124.

Keskitalo, K., Tuorila, H., Spector, T. D., Cherkas, L. F., Knaapila, A., Silventoinen, K., et al. (2007). Same genetic components underlie different measures of sweet taste preference. American Journal of Clinical Nutrition, 86, 1663-1669.

Kildegaard, H., Tønning, E., \& Thybo, A. K. (2011). Preference, liking and wanting for beverages in children aged 9-14 years. Role of sourness perception, chemical composition and background variables. Food Quality and Preference, 22, 620-627.

Lee, C. B., \& Lawless, H. T. (1991). Time-course of astringent material. Chemical Senses, 16, 225-238.
Liem, D. G., \& Mennella, J. A. (2003). Heightened sour preferences during childhood Chemical Senses, 28, 173-180.

Lim, J. Y., Urban, L., \& Green, B. G. (2008). Measures of individual differences in taste and creaminess perception. Chemical Senses, 33, 493-501.

Mattes, R. D. (1985). Gustation as a determinant of ingestion. Methological issues. The American Journal of Clinical Nutrition, 41, 672-683.

Mennella, J. A., Pepino, Y., \& Reed, D. R. (2005). Genetic and environmental determinants of bitter perception and sweet preferences. Pediatrics, 115 , 216-222.

Mitchell, M., Brunton, N. P., \& Wilkinson, M. G. (2013). The influence of salt taste threshold on acceptability and purchase intent of reformulated reduced sodium vegetable soups. Food Quality and Preference, 28, 356-360.

Mithril, C., Dragsted, L. O., Meyer, C., Blauert, E., Holt, M. K., \& Astrup, A. (2012). Guidelines for the New Nordic diet. Public Health Nutrition, 15, 1941-1947.

Moskowitz, H. R. (1994). Children versus adults. Food concepts and products. Just in time development (pp. 293-331). Trumbull, Connecticut, USA: Food and Nutrition press Inc.

Reed, D. R., Zhu, G., Breslin, P. A. S., Duke, F. F., Henders, A. K., Campbell, M. J., et al. (2010). The perception of quinine taste intensity is associated with common genetic variants in a bitter receptor cluster on chromosome 12. Human Molecular Genetics, 19, 4278-4285.

Rozin, P. (1976). The selection of food by rats, humans and other animals. In Advances in the Study of Behavior, J. Rosenblatt, R. A. Hinde, C. Beer, E. Shaw (Ed.), 6, 21-76, San Diego, CA: Academic.

Rozin, P., \& Vollmerck, T. A. (1986). Food likes and dislikes. Annual Review of Nutrition, 6, 433-456.

Steiner, J. E. (1974). Innate, discriminative human facial expressions to taste and smell stimulation. Annals of the New York Academy of Sciences, 237, 229-233.

Suomela, J. P., Vaarno, J., Sandell, M., Lehtonen, H., Tahvonen, R., Viikari, J., et al. (2012). Children's hedonic response to berry products. Effect of chemical composition of berries and hTAS2R38 genotype on liking. Food Chemistry, 135, 1210-1219.

Tang, X, Kälviäinen, N. \& Tuorila, H. (2001). Sensory and hedonic characteristics of juice of sea-buckthorn (Hippophaë rhamnoides L.) origins and hybrids. Vol. 34 Lebensmittel-Wissenschaft und - Technologie (pp. 102-110).

Tiitinen, K. M., Hakala, M. A., \& Kallio, H. P. (2005). Quality components of seabuckthorn (Hippophae rhamnoides) varieties. Journal of Agricultural and Food Chemistry, 53, 1692-1699. 